\title{
Review: glucosamine and chondroitin improve outcomes in osteoarthritis, but the magnitude of effect is unclear
}

\author{
McAlindon TE, La Valley MP, Gulin JP, et al. Glucosamine and chondroitin for treatment of osteoarthritis: a systematic \\ quality assessment and meta-analysis. JAMA 2000 Mar 15;283:1469-75.
}
QUESTION: In patients with osteoarthritis (OA), are glucosamine and chondroitin effective for relieving symptoms and improving function?

\section{Data sources}

Studies were identified by searching Medline (1966 to June 1999) and the Cochrane Controlled Trials Register and reviewing bibliographies of reviews and manuscripts. Meeting abstracts published in supplements of Arthritis and Rheumatism, the British Journal of Rheumatology, and Osteoarthritis and Cartilage (1978-98) were handsearched. Authors, content experts, and drug manufacturers were contacted.

\section{Study selection}

Studies in any language were selected if they were randomised, double blind, controlled trials that compared oral or parenteral glucosamine sulfate, glucosamine hydrochloride, or chondroitin sulfate with placebo for $\geq 4$ weeks in patients with OA of the knee or hip. Studies also had to include $\geq 1$ outcome measure from a list compiled by the reviewers.

\section{Data extraction}

2 reviewers assessed the quality of studies (14 item quality scale) and resolved disagreements by discussion. Data were extracted on patients, route of administration, joint with OA, outcomes, funding, allocation concealment, use of intention to treat analysis, and effect sizes.

\section{Main results}

17 studies met the inclusion criteria. 2 of these did not provide sufficient data for extraction and were excluded from the meta-analysis. The mean quality score was $36 \%$. 1 study reported adequate allocation concealment, and 1 used intention to treat analysis. No studies reported independent funding from any governmental or non-profit organisation. 6 studies of glucosamine, which involved 911 patients, had quality scores ranging from $12 \%$ to $52 \%$. Outcome measures were the Lequesne Index (a questionnaire based disability score) (3 studies), global pain scores (2 studies), and the Western Ontario and McMaster Universities Osteoarthritis Index score (1 study). Combined results showed a moderate benefit for glucosamine (effect size 0.44, 95\% CI 0.24 to 0.64 ).

9 studies of chondroitin, which involved 799 patients, had quality scores ranging from $14 \%$ to $55 \%$. Outcome measures were the Lequesne Index score (2 studies), global pain scores (5 studies), mobility scores (1 study), and use of non-steroidal anti-inflammatory drugs (1 study). Chondroitin had a large benefit (effect size 0.96, CI 0.63 to 1.3), but studies were heterogeneous $(\mathrm{p}<0.001)$. When the study with the largest effect size (4.56) was removed, heterogeneity was no longer significant (effect size 0.78, CI 0.60 to 0.95 ).

\section{Conclusions}

In patients with osteoarthritis, glucosamine and chondroitin are effective for improving outcomes. However, the magnitude of effect is unclear because of inconsistencies in study methods and dependence on industry support for study execution.

\section{COMMENTARY}

This systematic review by McAlindon et al evaluated the effect of glucosamine and chondroitin as symptom modifying drugs in OA. Although the authors conclude that these compounds may be useful for relieving pain and improving function in patients with $\mathrm{OA}$, they suggest that the magnitude of the effect of glucosamine or chondroitin might have been overestimated because of study design flaws and dependence on study sponsors. A previous metaanalysis that estimated the symptomatic effect of chondroitin sulfate for OA reached similar conclusions. ${ }^{1}$

The authors imply that the oldest studies tended to use poor methods. However, several trials have been presented or published in abstract form in recent months, ${ }^{2}$ and incorporating them into the meta-analysis might have reduced the heterogeneity of the results and given a better overview of the exact efficacy of chondroitin and glucosamine. Yet the message that more studies are needed before endorsing wide use of chondroitin and glucosamine in the treatment of patients with OA is well taken. It is particularly difficult to extrapolate the positive results obtained with pharmaceutical preparations to food supplements for which content, absorption, and metabolism are often doubtful.

Publication of the results from larger long term, prospective studies in the coming months should clarify the optimal dose and regimen of the drugs. The fact that such studies may be sponsored by manufacturers of either substance should not necessarily hinder their quality, provided that the results have been validated in randomised, double blind, controlled studies.

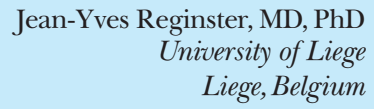

1 Leeb BF, Schweitzer H, Montag K, et al. A meta-analysis of chondroitin sulfate in the treatment of osteoarthritis. J Rheumatol 2000;27:205-11.

2 Reginster JY, Deroisy R, Paul I, et al. Glucosamine sulfate significantly reduces progression of the knee osteoarthritis over 3 years: a large randomised, placebo-controlled, doubleblind, prospective trial [abstract]. Arthritis Rheum 1999;42S:400A. 